\title{
Ropivacaína isolada e associada ao fentanil ou ao tramadol administrados pela via peridural em cães
}

\author{
Ropivacaine individually and in combination with fentanyl or tramadol, administered by \\ peridural via in dogs
}

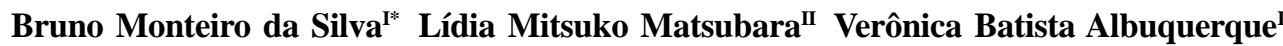 \\ Camila Aparecida de Almeida Maia ${ }^{I I I}$ Valéria Nobre Leal de Souza Oliva ${ }^{I V}$
}

RESUMO

A anestesia peridural é amplamente difundida no meio veterinário, utilizando-se o anestésico local isolado ou associado aos opióides, capazes de promover aumento do efeito analgésico. O objetivo deste estudo foi avaliar a função cardiorrespiratória e analgésica da ropivacaína isolada ou associada ao fentanil ou tramadol. Para tanto, oito cães foram tranqüilizados com acepromazina, submetidos à anestesia peridural com um dos seguintes protocolos: GR (ropivacaína), GRF (ropivacaína + fentanil), GRT (ropivacaína + tramadol), em volume total de $0,25 \mathrm{ml} \mathrm{kg}^{-1}$, e foram avaliados os parâmetros: freqüência cardíaca e respiratória, temperatura retal, pressão arterial sistólica, e gasometria do sangue arterial, os bloqueios sensitivo e motor, o grau de sedação e a ocorrência de possíveis efeitos indesejáveis. A diminuição da freqüência cardíaca nos grupos GRF e GRT foi mais intensa e ocorreu hipotermia significativa no GRF. Foi evidenciada sedação severa em GRF e GRT. O período de recuperação foi mais curto nos animais de GRT. O GRT foi o grupo que apresentou bloqueio mais cranial. Foram observadas bradicardia, hipotermia e síndrome de ShiffSherrington no período trans-anestésico em animais de todos os grupos. Nas 24 horas de período pós-anestésico, não foram evidenciados efeitos indesejáveis nos grupos. O GRF apresentou maior duração de anestesia e analgesia, enquanto que o GRT apresentou a menor duração de anestesia com analgesia intermediária e o GR apresentou duração intermediária, com menor analgesia. Não foram encontradas alterações respiratórias e hemogasométricas, porém, bradicardia, hipotermia e síndrome de Schiff-Sherrington, alterações trans-anestésicas comuns na anestesia peridural foram encontradas.

Palavras-chave: analgesia, epidural, extradural, opióides, sedação, bloqueio sensitivo e motor.

\begin{abstract}
Peridural anesthesia is broadly applied in the Veterinary field, using the isolated local anesthetic or in combination with opiates capable to increase the analgesic effect. This research compared analgesia and cardiorespiratory effects of epidural anaesthesia produced by ropivacaine alone or combined with fentanyl or tramadol in eight mixed breed adult dogs after sedation with acepromazina. Drugs were administered on the following protocols: GR (ropivacaine), GRF (ropivacaine + fentanyl), GRT (ropivacaine + tramadol), in $0.25 \mathrm{ml} \mathrm{kg}^{-1}$ of total volume. Heart and respiratory rate, rectal temperature, blood pressure and, gasometry of atrial blood were mensured, as well the sensory and motor blockade (latency and period of action), degree of sedation and side effects. The most important decrease of the heart rate occurred in GRF and GRT. Also significant hypothermia in GRF. Intense degree of sedation was observed in GRF and GRT. The period of recovery was shorter in GRT. The most cranial region of blockade occurred in GRT. Bradicardia, hypothermia and ShiffSherrington syndrom were observed in the transanesthetic period in animal from all of the groups. During 24 hours after the anaesthesia no side effects were observed. The GRF had the longer period of anesthesia and analgesia, GRT presented the shorter period of anesthesia with intermediate analgesia and, $G R$ presented intermediate period of anesthesia with lower degree of analgesia. Respiratory and hemogasometrics change were not found, but hipotermy, bradicardy and SchiffSherrington syndrome, side effects usually seen in peridural anesthesia, were observed in this study.
\end{abstract}

Key words: analgesia, epidural, extradural, opioids, sedation, sensory and motor blockade.

\footnotetext{
IPrograma de Pós-graduação em Ciência Animal, Universidade Estadual Paulista (UNESP), Araçatuba, SP, Brasil. E-mail: monteirobs@yahoo.com.br. *Endereço para correspondência: Rua Capitão Alfredo Cardoso, 152, 18031-030, Sorocaba, SP, Brasil.

IIPrograma de Pós-graduação em Anestesiologia Experimental, UNESP, Campus de Botucatu, Botucatu, SP, Brasil.

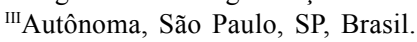

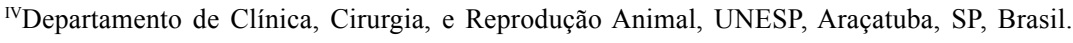




\section{INTRODUÇÃO}

As técnicas de bloqueio local constituemse em excelentes opções anestésicas, especialmente aos pacientes de maior risco (SKARDA, 2002). A anestesia peridural lombo-sacra, simples e segura, é a técnica regional mais utilizada para procedimentos retroumbilicais em cães (SKARDA, 2002; OTERO, 2005a). Apesar de ser considerada uma anestesia livre de complicações e com reduzidos riscos, animais debilitados podem não ser capazes de compensar possíveis alterações cardiovasculares, que seriam facilmente corrigidas em animais hígidos (INTELIZANO et al., 2002). Esta anestesia é também contra-indicada nos casos em que há infecção cutânea do local de punção, nas situações de hipovolemia ou desordens de coagulação, na ocorrência de doenças axonais centrais ou periféricas (JONES, 2001; INTELIZANO et al., 2002).

A ropivacaína é um anestésico local de duração de efeito longo, pertencente ao grupo das amino-amidas. Tem se apresentado como anestésico local efetivo no homem e em animais (FELDMAN et al., 1996). É comercializada apenas em sua fração $\mathrm{S}(-)$ (HURLEY et al., 1991; SIMONETTI, 1995), já que o isômero $\mathrm{R}(+)$ liga-se firmemente ao canal de sódio e é dissociado mais lentamente que o S(-), justificando a menor cardiotoxicidade da ropivacaína (SIMONETTI, 1995; CÔRTES et al., 2003). Os opióides são analgésicos eficazes e seguros, mas que podem levar à depressão respiratória, a distúrbios gastrintestinais e à sedação (FANTONI, 2002). O efeito analgésico e sedativo dos opióides dá-se pela ação em receptores específicos tanto no mesencéfalo(FANTONI, 2002; OTERO, 2005b) quanto na medula (EPIDURAL OPIATES, 1980; FANTONI, 2002), o que torna viável sua utilização nos bloqueios peridurais (EPIDURAL OPIATES, 1980), inclusive de maneira isolada. Ao utilizá-los por esta via, têm-se como vantagens a ausência de bloqueio simpático e a conseqüente não ocorrência de hipotensão, a facilidade na deambulação e a ausência de colapso cardiovascular ou convulsões (COUSINS \& MATHER, 1984). O fentanil é um opióide sintético, agonista $\mu$, de curto período de ação, com alta potência analgésica (FANTONI, 2002; OTERO, 2005b). Seu uso pela via peridural é bastante discutido, já que pela alta solubilidade e conseqüente permeabilidade facilitada pelas meninges, seu tempo de ação é reduzido, diminuindo sua potência no líquido cefalorraquidiano (JONES, 2001). O tramadol é um agonista $\mu$ que apresenta mecanismo de ação misto, com atividade bloqueadora monoaminérgica (GUEDES et al., 2002; INTELINZANO et al., 2002), o que o caracteriza como um opióide atípico. Sua utilização pela via peridural já é uma realidade na anestesia médica há algum tempo, porém, seu uso em medicina veterinária é recente (GUEDES et al., 2002).

O objetivo deste trabalho é avaliar a ropivacaína isolada ou em suas associações com o fentanil e o tramadol, em aplicação pela via peridural, no que se refere: a tempo de latência, a alterações cardiorrespiratórias, a tempo de bloqueio sensitivo e motor, à sedação, a alcance dos fármacos na medula, e a possíveis efeitos colaterais.

\section{MATERIAL E MÉTODOS}

O estudo foi realizado no laboratório experimental de cirurgia e anestesiologia da Universidade Estadual Paulista de Araçatuba. Foram utilizadas oito cadelas adultas, sem raça definida, com massa corporal entre 9,6 e $16 \mathrm{~kg}(13,2 \pm 0,7 \mathrm{~kg})$, sendo as mesmas submetidas a jejum hídrico de duas horas e alimentar de 12 horas. Todos os animais foram utilizados nos três protocolos anestésicos propostos, com intervalo mínimo de sete dias entre cada um. No início de cada procedimento, foram avaliados: freqüência cardíaca (FC), freqüência respiratória $(f)$ e temperatura retal $\left(\mathrm{T}^{\circ}\right)(\mathrm{M} 0)$. O protocolo utilizado como medicação pré-anestésica (MPA) foi a acepromazina, na dose de $0,05 \mathrm{mg} \mathrm{kg}^{-1}$ pela via intravenosa e, decorridos 15 minutos, foram repetidas as mensurações dos mesmos parâmetros (M1). Em seguida, foi realizado um botão anestésico com $0,2 \mathrm{ml}$ de ropivacaína no local de inserção da agulha para o bloqueio peridural. Após a contenção física, com o animal em decúbito esternal, foi realizado o bloqueio peridural. Uma agulha hipodérmica $40 \times 8$ que foi introduzida no espaço intervertebral lombo-sacro (L7-S1), com um dos protocolos: GR (Ropivacaína - 0,75\%), GRF (Ropivacaína - 0,75\% + Fentanil - $2 \mu \mathrm{g} \mathrm{kg}^{-1}$ ) ou GRT (Ropivacaína - 0,75\% + Tramadol - $2 \mathrm{mg} \mathrm{kg}^{-1}$ ), em um volume total de $0,25 \mathrm{ml} \mathrm{kg}^{-1}$. Em seguida, o animal foi mantido em decúbito dorsal (MAIA, 2006) por 15 minutos e, após este período, em decúbito lateral esquerdo para aferição dos parâmetros. Após a injeção peridural, a avaliação dos parâmetros e dos efeitos foi realizada a cada 20 minutos (M2 a M6) pelo tempo necessário para que todas as funções sensitivas e motoras iniciais fossem recuperadas (MF), sendo M7 o correspondente às médias aritméticas obtidas a partir dos 120 minutos da anestesia peridural até o momento em que o animal apresentou reflexo interdigital. A veia cefálica foi canulada, para administração de ringer com lactato na taxa de $5 \mathrm{ml} \mathrm{kg}^{-1} \mathrm{~h}^{-1}$, por meio de uma bomba de infusão contínua, durante todo o procedimento 
experimental. Os seguintes parâmetros foram avaliados nos momentos até que os animais se colocassem em posição quadrupedal: freqüência cardíaca $(\mathrm{FC})$, por meio da auscultação com estetoscópio; freqüência respiratória $(f)$, pela visualização dos movimentos respiratórios; temperatura retal $\left(\mathrm{T}^{\circ}\right)$, com um termômetro clinico digital; pressão arterial sistólica (PAS), aferida por meio de um "Doppler" ultra-sônico pelo método não-invasivo; gasometria arterial (antes da peridural, uma hora depois da realização e após recuperação do animal). O período de latência foi avaliado pelo relaxamento do esfíncter anal, pelo relaxamento de cauda e pela perda do reflexo interdigital. Além disso, foi observada a micção ou a defecação espontânea, após a aplicação da injeção peridural. A analgesia peridural foi verificada por meio da sonda de Foley 24 introduzida via retal até o cólon descendente, onde foi injetado água no cuff até o animal apresentar desconforto, volume este analisado individualmente, e de analgesia somática, por meio de eletroestimulação com uma descarga de $200 \mathrm{~V}$ e $30 \mathrm{~Hz} 20 \mathrm{~ms}^{-1}$ (NUNES, 2001) em eletrodos posicionados no subcutâneo da região de metatarsos direito. A duração de efeito da anestesia foi avaliada pela mensuração do tempo necessário para que o animal retornasse à posição quadrupedal com deambulação normal (bloqueio motor) e pelos tempos de duração do relaxamento do esfíncter anal, do relaxamento da cauda e da perda do reflexo interdigital. Observou-se, ainda, o grau de sedação utilizando-se os seguintes escores: 0: animal alerta, respondendo prontamente aos estímulos externos; 1: animal com sedação/tranqüilização leve - estado de relaxamento, calma e sonolência; 2: animal com sedação/tranqüilização moderada - estado de relaxamento e sonolência mais profundos, respondendo com dificuldade aos estímulos externos; 3: animal apresentando grau de sedação profunda (hipnose - transe que se assemelha ao sono) - alheio ao ambiente, mas podendo responder lentamente aos estímulos externos (MAIA, 2006). Também foi realizada a avaliação da resposta de panículo cutâneo pelo pinçamento da pele da região dorsal do animal e o acompanhamento, durante 24 horas, da ocorrência de possíveis efeitos adversos.

Os dados foram submetidos à análise de variância com medidas repetidas e as médias foram comparadas por meio do teste de Tukey. Para que os dados fossem submetidos à análise de variância, os mesmos foram testados quanto a sua normalidade e homogeneidade. Os valores das variáveis, da freqüência respiratória e do grau de sedação foram analisados usando o teste de Friedman seguido do teste de Dunn para comparações múltiplas. As variáveis: tempo de recuperação anestésica, tempo de latência e peso foram submetias à análise de variância e as médias foram comparadas por meio do teste de Tukey. Os valores das variáveis micção e defecção foram analisados usando o teste exato de Fisher. Todos os testes foram realizados em nível de significância de 5\%.

\section{RESULTADOS}

Em todos os grupos, houve redução da FC no decorrer do experimento, que foi mais acentuada $\mathrm{e}$ precoce nos grupos onde os opióides foram associados. A PAS reduziu-se no grupo da ropivacaína isolada a partir dos 80 minutos da realização da peridural (M5), mas não houve hipotensão em nenhum dos animais. A f e as variáveis hemogasométricas (pressão parcial de oxigênio e dióxido de carbono, $\mathrm{pH}$, bicarbonato e excesso de base) não apresentaram alterações. Durante o transanestésico, 58,3\% dos animais apresentaram hipotermia em algum dos momentos. Os animais de GR apresentaram um tempo menor em relação àqueles de GRF e de GRT para as variáveis: relaxamento de esfíncter anal e perda do reflexo interdigital (Tabela 1). Já o tempo de duração de efeito foi menor em GRT que nos demais grupos, enquanto que os animais do grupo GRF foram os que demoraram mais tempo para retornarem à posição quadrupedal (Tabela 2). Todos os animais, em todos os grupos, apresentaram sedação, porém, em GRT, a sedação foi significativamente mais severa e com menor duração que em GRF, enquanto que em GR a sedação foi moderada. $\mathrm{O}$ grupo GR mostrou menor variação na altura do bloqueio peridural, porém, em GRT e GRF, foram notadas as difusões mais craniais da anestesia. Durante o período anestésico, as alterações ocorridas em maior freqüência foram a bradicardia, seguida da hipotermia e da síndrome de Shiff-Sherrington. A taquicardia ocorreu em um animal do grupo GRF e, no grupo GR, dois animais apresentaram déficit proprioceptivo no membro pélvico esquerdo por um período de até 24 horas pós anestesia.

\section{DISCUSSÃO}

A redução da freqüência cardíaca ocorrida após a aplicação da acepromazina deve-se, provavelmente, à resposta compensatória aos efeitos alfa-adrenérgicos da mesma. Sabe-se que estes fármacos são capazes de levar a uma bradicardia sinusal, por bloqueios átrio-ventriculares e sinoatriais (CORTOPASSI et al., 2002b). Contrariando o descrito por ISHY (2001) e MAIA (2006), após a realização da peridural com anestésico local, houve diminuição da freqüência cardíaca, justificada pela possível ocorrência 
Tabela 1 - Média $(\overline{\mathrm{X}})$ e erro padrão da média (EPM) do período de latência, em minutos, de animais submetidos à anestesia peridural com ropivacaína (GR), ropivacaína associada ao fentanil (GRF) e ropivacaína associada ao tramadol (GRT).

\begin{tabular}{|c|c|c|c|}
\hline \multirow{2}{*}{ Variável } & \multicolumn{3}{|c|}{ 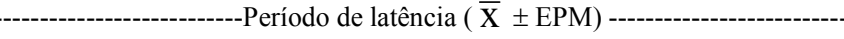 } \\
\hline & $\mathrm{R}$ & $\mathrm{RF}$ & $\mathrm{RT}$ \\
\hline Relaxamento do esfíncter anal & $2,1 \pm 0,2 \mathrm{~A}$ & $2,5 \pm 0,3 \mathrm{~A}$ & $3,1 \pm 0,6 \mathrm{~A}$ \\
\hline Relaxamento de cauda & $1,5 \pm 0,2 \mathrm{~A}$ & $1,5 \pm 0,2 \mathrm{~A}$ & $1,6 \pm 0,3 \mathrm{~A}$ \\
\hline Perda do reflexo interdigital & $4,3 \pm 0,6 \mathrm{~A}$ & $4,0 \pm 0,5 \mathrm{~A}$ & $6,0 \pm 0,9 \mathrm{~A}$ \\
\hline
\end{tabular}

Médias seguidas de mesma letra, na linha, não diferem entre si pelo teste de Tukey $(\mathrm{P}>0,05)$.

de bradicardia sinusal causada pelo bloqueio de fibras simpáticas, produzido pelos anestésicos locais.

Os fenotiazínicos, por produzirem efeitos calmantes nos animais (CORTOPASSI \& FANTONI, 2002a) e diminuírem a excitação, produziram redução da freqüência respiratória que, ao início do experimento, encontrava-se aumentada pela excitação dos animais. Estes valores mantiveram-se dentro de valores normais para a espécie durante todo o período anestésico.

A ocorrência de diminuição da temperatura retal pode ser justificada pelo uso do fenotiazínico, que é capaz de diminuir a temperatura corpórea (CORTOPASSI \& FANTONI, 2002a) pela vasodilatação, assim como por sua ação sobre o centro termorregulador no hipotálamo (BROCK, 1994; MAIA, 2006). Por outro lado, a aplicação peridural de anestésicos locais causa bloqueio simpático, levando à vasodilatação, que intensifica a hipotermia, como foi descrito por COUSINS \& MATHER (1984); SESSLER \& PONTE(1990); SKARDA (2002).

Não houve ocorrência significativa de hipotensão, já que os valores de pressão arterial sistólica (PAS) estiveram sempre em valores superiores a $80 \mathrm{mmHg}$, o que é considerado valor limite por DROBATZ \& LITTMAN (1997). No que se refere à alteração de pressão arterial, o presente estudo mostrou resultados divergentes dos descritos por MAIA (2006), que evidenciou hipotensão em 30,76\% dos animais estudados.

A pressão parcial de oxigênio $\left(\mathrm{PaO}_{2}\right)$ manteve-se dentro de valores normais para a espécie. A pressão parcial de dióxido de carbono $\left(\mathrm{PaCO}_{2}\right)$, o potencial hidrogênionico $(\mathrm{pH})$, o bicarbonato $\left(\mathrm{HCO}_{3}^{-}\right)$ e o excesso de base (BE) no sangue arterial não apresentaram diferenças estatísticas. Desta maneira, pode-se afirmar que não houve depressão respiratória.

O período de latência estabelecido para a ropivacaína (4,3 $\pm 0,6$ minutos) foi ligeiramente maior aos príodos descritos por FELDMAN et al.(1996) e por MAIA (2006), que obtiveram $2,9 \pm 0,8$ minutos e $3,5 \pm 0,5$ minutos respectivamente, e até mesmo maior que HURLEY et al.
(1991), que utilizaram a ropivacaína a 1\% com a adição de epinefrina e também encontraram valores inferiores $(3,4 \pm 0,9$ minutos $)$. Ao se avaliar a ocorrência de defecação e a micção involuntária após a realização da peridural, somente $12,5 \%$ dos animais apresentaram tal condição, e sem nenhum padrão relacionado aos grupos, diferindo de MAIA (2006), em que 25,6\% dos animais apresentaram micção involuntária.

Os animais anestesiados com ropivacaína isolada apresentaram sedação moderada durante a maior parte do período avaliado. Como não há relatos de sedação ou mecanismos de sedação com a utilização deste anestésico local, pode-se atribuir tal efeito à acepromazina. Os opióides aplicados pela via peridural são absorvidos sistemicamente, o que pode provocar sedação (FANTONI, 2002). Notou-se que em GRF o grau de sedação foi profundo durante a maior parte do experimento, tornando-se leve, em média, cinco horas e meia do início da anestesia. Na associação ao tramadol, um opióide de menor potência sedativa (FANTONI, 2002; OTERO, 2005b), os cães apresentaram um grau de sedação profundo, porém, de menor duração quando comparado à associação com fentanil na primeira hora de anestesia peridural, tornando-se, a partir de então, sedação de intensidade moderada, de maneira semelhante ao ocorrido com a utilização isolada da ropivacaína. Sendo assim, atribui-se a ocorrência de sedação nestes dois grupos ao efeito da acepromazina.

Os animais em que o fentanil foi utilizado apresentaram período de recuperação anestésica mais longo no que se refere à recuperação de tônus de cauda e do reflexo interdigital e à reversão da analgesia visceral e somática, assim como retomaram a posição quadrupedal mais precocemente. Tais resultados podem ser atribuídos à potencialização da ação do fentanil pela via peridural, como descrito por OTERO (2005b). Estes mesmos períodos foram significativamente mais curtos com a utilização de tramadol quando comparados aos dois outros grupos, o que poderia ser explicado pelo fato deste agente possuir potência menor do que a do fentanil, quando é 
Tabela 2 - Média $(\overline{\mathrm{X}})$ e erro padrão da média (EPM) do período de recuperação, em minutos, de animais submetidos à anestesia peridural com ropivacaína $(\mathrm{R})$, ropivacaína associada ao fentanil $(\mathrm{RF})$ e ropivacaína associada ao tramadol (RT).

\begin{tabular}{|c|c|c|c|}
\hline \multirow{2}{*}{ Variável } & \multicolumn{3}{|c|}{ 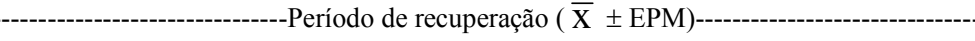 } \\
\hline & $\mathrm{R}$ & $\mathrm{RF}$ & RT \\
\hline Reflexo esfíncter anal & $91,4 \pm 19,2 \mathrm{~A}$ & $130,9 \pm 23,3 \mathrm{~A}$ & $137,9 \pm 21,2 \mathrm{~A}$ \\
\hline Tônus de cauda & $249,8 \pm 23,8 \mathrm{~A}$ & $233,6 \pm 17,9 \mathrm{~A}$ & $135,9 \pm 19,2 \mathrm{~B}$ \\
\hline Reflexo interdigital & $254,0 \pm 30,4 \mathrm{~A}$ & $241,9 \pm 26,0 \mathrm{AB}$ & $171,4 \pm 18,8 \mathrm{~B}$ \\
\hline Dor somática & $264,1 \pm 21,3 \mathrm{~A}$ & $269,5 \pm 28,3 \mathrm{~A}$ & $219,4 \pm 18,6 \mathrm{~A}$ \\
\hline Dor visceral & $207,7 \pm 40,3 \mathrm{~A}$ & $249,1 \pm 31,7 \mathrm{~A}$ & $177,6 \pm 22,9 \mathrm{~A}$ \\
\hline Posição quadrupedal & $294,1 \pm 26,9 \mathrm{AB}$ & $338,5 \pm 33,8 \mathrm{~A}$ & $234,5 \pm 16,4 \mathrm{~B}$ \\
\hline
\end{tabular}

Médias seguidas de mesma letra, na linha, não diferem entre si pelo teste de Tukey $(\mathrm{P}>0,05)$.

a ação sobre os receptores $\mu$ (OTERO, 2005b). Supõese, portanto, que o opióide acabou por "diluir" o anestésico local, diminuindo seu período de ação. A avaliação da sensibilidade de dor visceral monstrouse como uma forma de avaliação eficaz, já que $87,5 \%$ dos cães apresentaram resposta positiva, contestando assim o questionamento de MAIA (2006), no que diz respeito à funcionalidade deste teste. Tal questionamento deveu-se ao fato de que esse autor observou resposta positiva ao estímulo apenas em $23 \%$ dos animais, de maneira semelhante ao ocorrido nos estudos de GASPARINI (1999) e ISHY (2001), nos quais $26,6 \%$ e nenhum dos animais apresentaram resposta positiva ao estímulo, respectivamente. Este estudo permitiu verificar grande variação individual nos volumes a serem injetados no cuff da sonda de Foley (de 25 a $55 \mathrm{ml}$ ), o que deve ser determinado individualmente antes de submeter o animal à anestesia o que, caso não seja feito, pode levar a resultados falhos quando volumes iguais forem utilizados em todos os animais. No presente trabalho, foram encontradas algumas alterações trans-anestésicas, que, segundo OTERO (2005a), estão relacionadas principalmente à progressão cranial do bloqueio, que reflete no equilíbrio hemodinâmico, seja pelo bloqueio simpático ou pela absorção dos fármacos do espaço peridural à circulação sistêmica. A síndrome de Schiff-Sherrington, observada quando são bloqueadas fibras na região tóraco-lombar, foi encontrada em $25 \%$ dos animais estudados, o que difere dos valores encontrados por MAIA (2006), em que esta alteração ocorreu em apenas 7,5\% dos animais estudados sendo, contudo, inferior ao observado por CAMPAGNOL et al. (2002), que evidenciaram a ocorrência desta síndrome em $66,6 \%$ e em $16,6 \%$ dos animais após a utilização lidocaína/clonidina ou de lidocaína/tramadol pela via peridural, respectivamente. Outra alteração observada foi a ocorrência de déficit proprioceptivo do membro pélvico esquerdo, que provavelmente deveu-se ao maior depósito do fármaco do lado esquerdo do espaço peridural já que, após 15 minutos da realização da injeção peridural, os animais foram colocados neste decúbito lateral para avaliação. Decorridas 24 horas da realização do procedimento, todos os animais, incluindo aqueles nos quais os efeitos indesejáveis ocorreram, encontravam-se em completo estado de normalidade, comprovando que tais ocorrências são passageiras e não comprometem a saúde do animal.

\section{CONCLUSÕES}

Concluiu-se que a utilização de ropivacaína pela via peridural em cães, na forma isolada ou associada ao tramadol ou ao fentanil, é uma prática segura, não produzindo alterações respiratórias ou desequilíbrio ácido-básico significativos, podendo-se esperar, contudo, a ocorrência de alguns efeitos colaterais passageiros como bradicardia, hipotermia e síndrome de Schiff-Sherrington. A associação ao tramadol aumenta o período de latência e associação ao fentanil, prolonga o efeito da anestesia peridural, podendo produzir até aproximadamente quatro horas de analgesia e de cinco a seis horas de decúbito em cães.

\section{COMITÊ DE ÉTICA E BIOSSEGURANÇA}

O projeto experimental foi aprovado pela Comissão de Ética em Experimentação Animal (CEEA) da Unesp - Araçatuba, registrado sob o número 06/05.

\section{AGRADECIMENTOS}

Agradecimentos à Bayer, pela doação de Advantages Max $3^{\circledR}$, e à Fort Dodge, pela doação das vacinas Duramune $^{\mathbb{R}}$.

Ciência Rural, v.38, n.8, nov, 2008. 


\section{REFERÊNCIAS}

BROCK N. Acepromazina revised. Canadian Veterinary Journal, v.35, n.6, p.458-459, 1994.

CAMPAGNOL, D. et al. Efeitos cardiorrespiratório e analgésico da clonidina e do tramadol associados a lidocaína, em anestesia peridural de cães. Revista Brasileira de Ciência Veterinária, v.9, n.1, p.286-288, 2002.

CÔRTES, C.A.F. et al. Estudo comparative entre bupivacaína a $0,5 \%$, mistura enantiométrica de bupivacaína (S75-R25) a $0,5 \%$ e ropivacaína a $0,75 \%$ associadas ao fentanil em anestesia peridural para cesarianas. Revista Brasileira de Anestesiologia, v.53, n.2, p.177-187, 2003.

CORTOPASSI, S.R.G.; FANTONI D.T. Medicação préanestésica. In: FANTONI, D.T.; CORTOPASSI, S.R.G. Anestesia em cães e gatos. São Paulo: Roca, 2002a. Cap.13, p.151-158.

CORTOPASSI, S.R.G. et al. Complicações da anestesia. In: FAnTONi, D.T.; CORTOPASSi, S.R.G. Anestesia em cães e gatos. São Paulo: Roca, 2002b. Cap.33, p. 347-361.

COUSINS, M.J.; MATHER, M. Intrathecal and epidural administration of opioids. Anesthesiology, v.61, n.3, p.276$310,1984$.

DROBATZ, K.J.; LITTMAN, M.P. Distúrbios hipertensivos e hipotensivos. In: ETTINGER, S.J.; FELDMAN, E.C. Tratado de medicina interna veterinária. 4.ed. São Paulo: Manole, 1997. 2v. V.1, cap.19, p.126-135.

EPIDURAL OPIATES. Lancet, v.1, n.8175, p.962-967, 1980.

FANTONI, D.T. Fisiopatologia e controle da dor. In: FANTONI, D.T.; CORTOPASSI, S.R.G. Anestesia em cães e gatos. São Paulo: Roca, 2002. Cap.31, p.323-336.

FELDMAN, H.S. et al. Antinociceptive and motor-blocking efficacy of ropivacaine and bupivacaine after epidural administration in the dog. Regional Anesthesia, v.21, n.4, p.318-325, 1996.

GASPARINI S.S. Efeito cardiorrespiratório e analgésico somático e visceral da anestesia epidural com lidocaína, ropivacaína ou a associação de lidocaína e xilazina em cães. 1999. 51f. Dissertação (Mestrado em Cirurgia Veterinária) - Faculdade de Medicina Veterinária e Zootecnia, Universidade Estadual Paulista, Botucatu.
GUEDES, A.G.P. et al. Tramadol via peridural em cães submetidos à substituição do ligamento cruzado cranial. Ciência Rural, v.32, n.2, p.345-346, 2002.

HURLEY, R.J, et al. The effects of epinephrine on the anesthetic and hemodynamic properties of ropivacaine and bupivacaine after epidural administration in the dog. Regional Anesthesia. v.16, p.303-308, 1991.

INTELIZANO, T.R. et al. Técnicas de anestesia local. In: FANTONI, D.T.; CORTOPASSI, S.R.G. Anestesia em cães e gatos. São Paulo: Roca, 2002. Cap.19, p.199-208.

ISHY, H.M. Uso da lidocaína isolada ou associada à quetamina ou ao butorfanol, em anestesia epidural em cães: avaliação cardiorrespiratória e analgésica. 2001. 79f. Dissertação (Mestrado em Anestesiologia Experimental) Faculdade de Medicina, Universidade Estadual Paulista, Botucatu.

JONES, R.S. Epidural analgesia in the dog and cat. Veterinary Journal, v.161, n.2, p.123-131, 2001.

MAIA, C.A.A. Avaliação cardiorrespiratória e analgésica da ropivacaína associada à morfina, petidina ou butorfanol administrados pela via peridural em cães. 2006. 114f. Dissertação (Mestrado em Anestesiologia Experimental) - Faculdade de Medicina, Universidade Estadual Paulista, Botucatu.

NUNES, N. et al. Efeitos da associação fentanil e dopreridol na concentração alveolar mínima do desflurano, em cães. Ars Veterinária, Jaboticabal, SP, v.17, n.2, p.86-92, 2001.

OTERO, P. Administração epidural e espinhal de analgésicos. In: _____. Dor: avaliação e tratamento em pequenos animais. São Caetano do Sul: Interbook; 2005a. Cap.14, p. 192-211.

OTERO, P. Drogas analgésicas. In:___ Dor: avaliação e tratamento em pequenos animais. São Caetano do Sul: Interbook; 2005b. Cap.7, p.96-109.

SESSLER, D.I.; PONTE, J. Shevering during epidural anesthesia. Anesthesiology, v.72, p.816-821, 1990.

SIMONETTI, M.P.B. Ropivacaína: estado atual e perspectivas futuras. Revista Brasileira de Anestesiologia, v.45, n.2, p.131-140, 1995.

SKARDA, R.T. Local and regional anesthesic and analgesic techiniques: dogs. In: THURMON, J.C. et al. Lumb \& Jones. Veterinary anesthesia. 3.ed. São Paulo: Roca, 2002. Cap.16, p.426-447. 